\title{
Cholesterol lowering drug use and breast cancer survival: the Multiethnic Cohort Study
}

\author{
Nafeesa Moksud ${ }^{1}$ (1) - Lenora W. M. Loo ${ }^{2}$. Juan Yang ${ }^{1} \cdot$ Chiung-Yu Huang $^{1} \cdot$ Christopher A. Haiman $^{4}$. \\ Loïc Le Marchand ${ }^{3} \cdot$ Lynne R. Wilkens $^{3} \cdot$ Iona Cheng $^{1}$
}

Received: 1 June 2021 / Accepted: 8 August 2021 / Published online: 30 August 2021

(c) The Author(s) 2021

\begin{abstract}
Purpose Prior studies conducted primarily in white populations have suggested that pre-diagnostic cholesterol lowering drugs (CLDs) improved survival among women with breast cancer (BC). However, this association had not been well characterized in diverse racial/ethnic populations. We investigated whether pre-diagnostic CLD use is associated with all-cause and BC-specific mortality among female BC cases of the Multiethnic Cohort (MEC).

Methods CLD use was ascertained through questionnaires administered in 2003-2008. A total of 1448 incident BC cases were identified by linkage to SEER cancer registries in Hawaii and California from 2003 to 2014. Multivariable Cox regression was conducted to estimate hazard ratios (HR) and 95\% confidence intervals (CI) of the associations of pre-diagnostic CLD use with all-cause and BC-specific mortality, adjusting for tumor characteristics, first course of treatment, health behaviors, co-morbidities, and demographics. Subgroup analyses by stage and hormone receptor status were conducted for all-cause mortality.

Results There were 224 all-cause and $87 \mathrm{BC}$-specific deaths among the $1448 \mathrm{BC}$ cases during a median follow-up of 4.5 years after diagnosis. Women with BC who ever used CLDs had a $27 \%$ lower hazard of all-cause mortality (HR $0.73,95 \%$ CI 0.54-0.98) and 17\% lower hazard of BC-specific mortality (HR 0.83, 95\% CI 0.49-1.39) compared to never users. CLD use reduced mortality among women with advanced-stage tumors and hormone receptor-positive breast tumors (HR $0.5495 \%$ CI 0.33-0.90; HR 0.69, 95\% CI 0.48-0.99, respectively).

Conclusion These findings demonstrate an improved survival associated with CLD use prior to diagnosis in a multiethnic population of women with $\mathrm{BC}$.
\end{abstract}

Keywords Breast cancer $\cdot$ Survival $\cdot$ Mortality $\cdot$ Cholesterol lowering drug $\cdot$ Multiethnic Cohort

$\begin{array}{ll}\text { Abbreviations } \\ \text { CLD/CLDs } & \text { Cholesterol lowering drug(s) } \\ \text { BC } & \text { Breast cancer } \\ \text { MEC } & \text { Multiethnic Cohort }\end{array}$

Iona Cheng

iona.cheng@ucsf.edu

1 Department of Epidemiology and Biostatistics, University of California, San Francisco, 550 16th Street, San Francisco, CA 94158, USA

2 Cancer Biology Program, University of Hawaii Cancer Center, Honolulu, HI, USA

3 Population Sciences in the Pacific Program, University of Hawaii Cancer Center, Honolulu, HI, USA

4 Department of Preventive Medicine, Keck School of Medicine, University of Southern California, Los Angeles, CA, USA

\begin{tabular}{|c|c|}
\hline HR & Hazard ratio \\
\hline $\mathrm{CI}$ & Confidence interval \\
\hline HMG Co-A & $\begin{array}{l}\text { 3-Hydroxy-3-methyl-glutaryl-coenzyme A } \\
\text { reductase }\end{array}$ \\
\hline QX3 & MEC Questionnaire 3 \\
\hline SEER & $\begin{array}{l}\text { Surveillance, Epidemiology, and End } \\
\text { Results Program }\end{array}$ \\
\hline AHEI & Alternate Healthy Eating Index \\
\hline ER & Estrogen receptor \\
\hline PR & Progesterone receptor \\
\hline HER-2 & Human epidermal growth factor receptor 2 \\
\hline
\end{tabular}




\section{Introduction}

Breast cancer is the most commonly diagnosed cancer among women in the United States, and advances in disease management and the promotion of breast cancer screening have improved the survival rates of breast cancer. Recent endeavors in re-purposing old drugs for novel uses [1-4] sparked interest in investigating the effects of commonly used medications on health outcomes in cancer survivors [5-7]. Cholesterol lowering drugs are a good candidate as they are commonly prescribed amongst the general population. Additionally, some cholesterol lowering drugs such as statins (hydroxymethylglutaryl-CoA (HMG-CoA) reductase inhibitors) were discovered to have oncotherapeutic potential in breast cancer cell lines [8]. While the various purported mechanisms of how statins have anti-tumor properties are reported extensively elsewhere $[9,10]$, the most commonly reported mechanism is that statins inhibit the rate limiting step in the mevalonate pathway, preventing the synthesis of essential metabolites for multiple cellular processes, including cellular proliferation, migration, and survival [11-14].

Several epidemiologic cohort studies have shown that use of cholesterol lowering drugs confers benefit on allcause and breast cancer-specific mortality among breast cancer cases [15-20]. In the largest cohort study to date, conducted among 20,559 Swedish women with breast cancer, Borgquist et al. reported all-cause mortality hazard ratios of 0.76 (95\% CI 0.66-0.88) and 0.89 (95\% CI $0.82-0.96$ ) for pre-and post-diagnostic statin use, respectively, compared to never users. The HRs for breast cancerspecific mortality was 0.77 (95\% CI $0.63-0.95)$ and 0.83 (95\% CI 0.75-0.93) for pre- and post-diagnostic statin use, respectively, compared to never users [19]. The majority of studies on the association between statins and breast cancer outcomes were conducted among cohorts of Scandinavian and Western European women [15-19, 21, 22].

Two U.S. studies [23-25] with substantial non-white breast cancer cases did not report race/ethnicity-specific findings. Li et al. [25] reported findings on a cohort of 1523 women with breast cancer (60\% white, $31 \%$ African American, $8 \%$ other breast cancer cases) with all-cause mortality hazards of 0.38 (95\% CI $0.17-0.85$ ) for women who had used statins for 5 years or longer. Shaitelman et al. [24] reported all-cause mortality hazards of 0.74 (95\% CI 0.20-2.77) and breast cancer-specific mortality hazards of 0.70 (95\% CI $0.47-1.03)$ in a cohort of 869 women with triple negative breast cancer $(60 \%$ white, $22 \%$ African American, 14\% Hispanic, 5\% other). Another study by Leiter et al. [23] examined racial differences in breast cancer prognosis using the Nottingham Prognostic Index in 100 African American and 487 white breast cancer cases. Leiter et al. reported that pre-diagnostic statin use could not explain the better prognosis for white breast cancer cases in comparison to African American cases [23]. As there have been limited studies conducted among diverse racial/ethnic groups, the objective of this report was to examine the association between use of cholesterol lowering drugs prior to diagnosis and mortality in a multiethnic population of breast cancer cases.

\section{Methods}

The Multiethnic Cohort (MEC) Study was established to investigate diet and lifestyle factors and cancer in five racial/ ethnic groups-African American, Japanese Americans, Latino American, Native Hawaiian and white [26]. Participants were recruited from a population-based sampling frame, mainly using driver's license records from Hawaii and Southern California. Participants were enrolled into the cohort in 1993 through 1996 by completing a baseline selfadministered questionnaire that surveyed demographics, body size, personal and family medical history, occupational history, food intake, physical activity, and medication use. This questionnaire with minor modifications was repeated in 2003-2008 (Qx3). Further details on the design, implementation, and composition of the MEC study are described elsewhere [26].

Use of cholesterol lowering drug use was not included at baseline, as use was not common at that time, but was added at follow-up questionnaire (QX3). The following question at QX3 was asked to assess cholesterol lowering drug use: Have you ever taken any of the following medications at least two times per week (for 1 month or longer)? The question was posed for high cholesterol medication, with examples of brand names provided (Lipitor, Mevacor, Zocor, Pravachol, Lopid or other). Participants responded "no," "yes, but not at this time," or "yes, currently." If participants answered "yes," they were asked to mark the length of medication use as 1 year or less, 2-3 years, 4-5 years, 6-10 years, or 11 years or more.

Cancer cases in the MEC were identified via linkages to Hawaii and California cancer registries both members of National Cancer Institute Surveillance, Epidemiology, and End Results Program (SEER) Program. Information on date of diagnosis, tumor characteristics (SEER summary stage of disease, hormone receptor status, lymph node involvement), and first course of treatment (surgery, hormone therapy) were obtained from the registries. Vital status and cause of death were collected through state death certificates and linkage with the National Death Index. For this study, eligible MEC women were considered only if they had completed QX3 (sent and collected from 2003 to 2008), had no breast cancer diagnosis prior to QX3, and had an invasive 
breast cancer diagnosis after receipt of QX3 through 2014. We excluded breast cancer cases, who did not provide data on cholesterol lowering medication use $(n=121)$, premenopausal women $(n=7)$ or women with unknown menopausal status $(n=4)$, resulting in a final study population of 1448 breast cancer cases with cholesterol lowering drug use ascertained prior to diagnosis. These women were followed for vital status from date of diagnosis (2003-2014) to study end date (31st December 2014), with a median follow-up time of 4.5 years $(\mathrm{SD} \pm 2.86$ years).

\section{Statistical analysis}

We used chi-square and Student's $t$-tests to evaluate differences in study characteristics by pre-diagnostic cholesterollowering drug use. Multivariable-adjusted hazard ratios (HRs) and 95\% confidence intervals (CI) for all-cause mortality were estimated using Cox proportional hazards regression, using age as the time metric. Follow-up started at the age of breast cancer diagnosis and ended at the age of death or age at end of follow-up (12/31/2014). Models were adjusted for race/ethnicity (African American, Japanese Americans, Latino American, Native Hawaiian, and white), level of education (high school graduate or less, college or vocational school, graduate or professional school), and the following covariates collected at QX3: body mass index (normal, underweight, overweight, obese class I, obese class II/III, unknown), daily energy intake $(<1164 \mathrm{cal}$, 1164-1550 cal, 1550-2037 cal, > $2037 \mathrm{cal})$, Alternate Healthy Eating Index (AHEI) Score [27] (27-60, 60-67, $67-74,74-101)$, age at menarche $(<11$ years, $11-12$ years, 13-14 years, 15-16 years, $\geq 17$ years, unknown), cardiovascular disease risk (none, hypertensive or taking hypertension medications, history of cardiovascular disease or stroke), diabetes (not diabetic, diabetic), tumor stage at diagnosis (localized, regional, distant, unknown), lymph node status at diagnosis (lymph node negative, 1 or more lymph node positive, unknown), estrogen and progesterone receptor (ER and $\mathrm{PR})$ status $(\mathrm{ER}+\mathrm{PR}+, \mathrm{ER}+\mathrm{PR}-, \mathrm{ER}-\mathrm{PR}+, \mathrm{ER}-\mathrm{PR}-$, unknown), surgery (no, yes), and hormone therapy (no, yes). Chemotherapy and mammography screening were found not to be associated with mortality among this sample and were not included as covariates. Cholesterol lowering drug use was categorized as "never", "past", and "current". "Past" and "current" users were also combined to make an "ever" user category. Duration of cholesterol lowering drug use was categorized into "less than or equal to 3 years of use" and "more than 3 years of use". Subgroup analysis was conducted by race/ethnicity, tumor stage (localized; advanced: regional and distant), and hormone receptor status (hormone receptor positive: ER+ and/or PR+; hormone receptor negative: ER- and PR-). We assessed heterogeneity of associations for cholesterol lowering drug use and all-cause mortality by race/ethnicity, stage, and hormone receptor status using a global test of interaction. Multivariable adjusted hazard ratios were also calculated for breast-cancer specific mortality. The proportional hazards assumption was assessed by examining $\log (-\log$ (survival function)) plots for diverging or crossing survival curves over time and by assessing the Schoenfeld residuals. To explore racial/ethnic differences among breast cancer cases, we evaluated cholesterol lowering drug use prevalence, demographics, lifestyle factors, comorbidities, and treatment factors across the five racial/ethnic groups. In addition, we applied the Inverse Probability of Treatment Weighting [28] approach to allcause and breast-cancer specific models of ever versus never cholesterol lowering drug use. The weight was determined for each individual as $w=Z / e+((1-Z)) /(1-e)$, where $Z$ is the indicator variable denoting whether CLD was used or not; and e denoting the predicated probability of cholesterol lowering drug use (ever versus never) conditioned on all other covariates adjusted for in the original multivariable Cox model. SAS version 9.4 (SAS Institute Inc., Cary, NC) and Stata 16.1 (College Station, TX: StataCorp LLC.) were used for analyses with a two-sided $p$-value of $<0.05$ considered to be statistically significant.

\section{Results}

Our final study population consisted of 1448 breast cancer cases $(54.8 \%$ never users, $6.7 \%$ past users, and $38.5 \%$ current users of cholesterol lowering drugs). During the median follow-up time of 4.5 years after diagnosis, 224 cases died, of which 87 deaths were due to breast cancer. The median follow-up time did not differ significantly by cholesterol lowering drug use ( 4.52 years for ever users, 4.47 years for never users). We observed racial/ethnic differences between use of cholesterol lowering drugs among our breast cancer cases (Table 1). Among ever users, the highest percentage of use was seen among Japanese American cases (55.9\%) followed by Native Hawaiian (52.3\%), Latino American (44.7\%), African American (39.4\%), and white (36.4\%) cases. Cholesterol lowering drug use was more likely in older or diabetic cases, those with higher BMIs and higher cardiovascular risk, and those receiving hormonal therapy (Table 1).

Compared to never-users, breast cancer cases who reported having ever used cholesterol lowering drugs prior to diagnosis had a $27 \%$ lowered hazard of all-cause mortality (HR 0.73, 95\% CI 0.54-0.98, Table 2). A similar pattern of association was observed across race/ethnicity with the exception of white women with breast cancer (Supplementary Table $1 ; p$ for heterogeneity by race/ethnicity $=0.11$ ).

Lower mortality was seen for current users (HR 0.72 , 95\% CI 0.53-0.99) and suggested for past users (HR 0.75, 
Table 1 Study characteristics of female breast cancer cases in the MEC diagnosed between 2003 and 2014 by cholesterol lowering drug use

\begin{tabular}{|c|c|c|c|c|c|}
\hline \multirow{2}{*}{$\overline{\text { Demographics }^{a}}$} & \multicolumn{2}{|c|}{ Never user $(n=793)$} & \multicolumn{2}{|c|}{ Ever user $(n=655)$} & \multirow[t]{2}{*}{$p$ value } \\
\hline & & & & & \\
\hline Age at diagnosis, mean $\pm \mathrm{SD}$, year & 72.9 & \pm 8.24 & 74.5 & \pm 7.95 & $\leq 0.001$ \\
\hline Years of follow-up, mean $\pm S D$, year & 4.47 & \pm 2.82 & 4.52 & \pm 2.90 & 0.73 \\
\hline Race/Ethnicity, $n, \%$ & & & & & 0.001 \\
\hline African American & 120 & $60.6 \%$ & 78 & $39.4 \%$ & \\
\hline Japanese American & 224 & $44.1 \%$ & 246 & $55.9 \%$ & \\
\hline Latino American & 115 & $55.3 \%$ & 93 & $44.7 \%$ & \\
\hline Native Hawaiian & 67 & $47.7 \%$ & 85 & $52.3 \%$ & \\
\hline White & 267 & $63.6 \%$ & 153 & $36.4 \%$ & \\
\hline *Marital status, $n, \%$ & & & & & 0.13 \\
\hline Married & 418 & $53.7 \%$ & 360 & $46.3 \%$ & \\
\hline Separated/Divorced & 140 & $59.3 \%$ & 96 & $40.7 \%$ & \\
\hline Widowed & 161 & $52.4 \%$ & 146 & $47.6 \%$ & \\
\hline Never married & 63 & $63.0 \%$ & 37 & $37.0 \%$ & \\
\hline *Level of education, $n, \%$ & & & & & $\mathbf{0 . 0 3}$ \\
\hline High school graduate or less & 249 & $50.1 \%$ & 248 & $49.9 \%$ & \\
\hline College or vocational school & 390 & $56.4 \%$ & 301 & $43.6 \%$ & \\
\hline Graduate/professional school & 146 & $59.1 \%$ & 101 & $40.9 \%$ & \\
\hline \multicolumn{6}{|l|}{ Health behaviors and past medical history ${ }^{1}$} \\
\hline Body Mass Index, $\mathrm{kg} / \mathrm{m}^{2}$, mean $+\mathrm{SD}$ & 26.7 & \pm 6.01 & 28.3 & \pm 6.26 & $\leq 0.001$ \\
\hline Daily caloric intake, kcal, mean +SD & 1682 & \pm 718 & 1679 & \pm 784 & 0.96 \\
\hline Alternate Healthy Eating Index Score, mean + SD & 67.0 & \pm 10.20 & 66.7 & \pm 8.96 & 0.51 \\
\hline *Age at menarche, $n, \%$ & & & & & 0.12 \\
\hline$<11$ years & 58 & $45.3 \%$ & 70 & $54.7 \%$ & \\
\hline $11-12$ years & 346 & $53.9 \%$ & 296 & $46.1 \%$ & \\
\hline $13-14$ years & 304 & $58.2 \%$ & 218 & $41.8 \%$ & \\
\hline $15-16$ years & 73 & $55.3 \%$ & 59 & $44.7 \%$ & \\
\hline$>17$ years & 5 & $55.6 \%$ & 4 & $44.4 \%$ & \\
\hline Cardiovascular disease, $n, \%$ & & & & & $\leq \mathbf{0 . 0 0 1}$ \\
\hline None & 319 & $73.5 \%$ & 115 & $26.5 \%$ & \\
\hline Hypertensive, or taking hypertension medications & 409 & $49.3 \%$ & 421 & $50.7 \%$ & \\
\hline History of cardiovascular disease or stroke & 65 & $35.3 \%$ & 119 & $64.7 \%$ & \\
\hline Diabetes, $n, \%$ & & & & & $\leq \mathbf{0 . 0 0 1}$ \\
\hline Not diabetic & 718 & $60.3 \%$ & 473 & $39.7 \%$ & \\
\hline Diabetic & 75 & $29.2 \%$ & 182 & $70.8 \%$ & \\
\hline \multicolumn{6}{|l|}{ Tumor characteristics ${ }^{b}$} \\
\hline$*$ Stage at diagnosis, $n, \%$ & & & & & 0.52 \\
\hline Localized & 578 & $54.2 \%$ & 488 & $45.8 \%$ & \\
\hline Regional & 181 & $56.4 \%$ & 140 & $43.6 \%$ & \\
\hline Distant & 26 & $61.9 \%$ & 16 & $38.1 \%$ & \\
\hline *Lymph node status at diagnosis, $n, \%$ & & & & & 0.37 \\
\hline All nodes negative & 588 & $53.9 \%$ & 503 & $46.1 \%$ & \\
\hline 1 or more nodes positive & 176 & $56.8 \%$ & 134 & $43.2 \%$ & \\
\hline *Estrogen and progesterone receptor status, $n, \%$ & & & & & 0.13 \\
\hline $\mathrm{ER}+/ \mathrm{PR}+$ & 544 & $54.0 \%$ & 464 & $46.0 \%$ & \\
\hline $\mathrm{ER}+/ \mathrm{PR}-$ & 109 & $60.2 \%$ & 72 & $39.8 \%$ & \\
\hline $\mathrm{ER}-/ \mathrm{PR}+$ & 3 & $27.3 \%$ & 8 & $72.7 \%$ & \\
\hline $\mathrm{ER}-/ \mathrm{PR}-$ & 114 & $57.0 \%$ & 86 & $43.0 \%$ & \\
\hline *Surgery, $n, \%$ & & & & & 0.75 \\
\hline No & 37 & $52.9 \%$ & 33 & $47.1 \%$ & \\
\hline Yes & 748 & $54.8 \%$ & 616 & $45.2 \%$ & \\
\hline
\end{tabular}


Table 1 (continued)

\begin{tabular}{llllll}
\hline & \multicolumn{2}{c}{ Never user $(n=793)$} & Ever user $(n=655)$ & $p$ value \\
\hline *Hormone therapy, $n, \%$ & & & & \multicolumn{2}{c}{0.03} \\
No & 370 & $57.5 \%$ & 274 & $42.5 \%$ & \\
Yes & 376 & $51.6 \%$ & 352 & $48.4 \%$ & \\
\hline
\end{tabular}

$p$-values provided by using chi-squared and Student's $t$-tests. Bolded values indicate $p$-values $\leq 0.05$

$S D$ standard deviation, $E R$ estrogen receptor, $P R$ progesterone receptor

*Counts in these categories may not add up to totals due to participants with missing information

${ }^{a}$ Demographics, health behavior and past medical history, and cholesterol lowering drug use was collected from MEC Qx3 administered between 2003 and 2008

${ }^{\mathrm{b}}$ Tumor data was collected at time of diagnosis (2003-2014)

Table 2 Association between cholesterol lowering drug use and all-cause mortality among women diagnosed with breast cancer in the MEC (2003-2014)

\begin{tabular}{llllll}
\hline Cholesterol lowering drug use & $n$ Cases & $n$ Deaths & HR & $95 \%$ CI & $p$ value \\
\hline Never/Ever & & & & & \\
$\quad$ Never (reference) & 793 & 123 & 1.00 & & $\mathbf{0 . 0 4}$ \\
$\quad$ Ever & 655 & 101 & $\mathbf{0 . 7 3}$ & $\mathbf{0 . 5 4 - 0 . 9 8}$ & $\mathbf{0 . 0 4}$ \\
Never/current/past & & & & & \\
Never (reference) & 793 & 123 & 1.00 & & 0.29 \\
Past & 97 & 19 & 0.75 & $0.43-1.28$ & $\mathbf{0 . 0 5}$ \\
Current & 558 & 82 & $\mathbf{0 . 7 2}$ & $\mathbf{0 . 5 3 - 0 . 9 9}$ & \\
Duration of use & & & & & $\mathbf{0 . 0 1}$ \\
Never (reference) & 793 & 123 & 1.00 & & 0.57 \\
3 years of use or less & 320 & 50 & $\mathbf{0 . 6 2}$ & $\mathbf{0 . 4 2 - 0 . 9 1}$ & \\
More than 3 years of use & 309 & 49 & 0.90 & $0.62-1.29$ & 0.50 \\
\hline
\end{tabular}

Adjusted for age at breast cancer diagnosis, race/ethnicity, level of education, body mass index, daily caloric intake, Alternate Healthy Index Score, age at menarche, cardiovascular disease, diabetes, tumor stage, lymph node status, hormone receptor status, surgery, and hormone therapy

Bolded values indicate $p$-values $\leq 0.05$
95\% CI 0.43-1.28) in comparison to never-users (Table 2). There was no significant difference in the association between past users and current users $(p=0.34)$. In addition, cholesterol-lowering drug use for less than or equal to 3 years was associated with lower all-cause mortality in comparison to never use (HR 0.62, 95\% CI 0.42-0.91, Table 2). No significant difference in association was found between breast cancer cases that used cholesterol lowering drugs for 3 years or less versus more than 3 years $(p=0.34)$. For breast cancer-specific mortality, breast cancer cases who reported having ever versus never used cholesterol lowering drugs had a $17 \%$ lowered hazard of mortality (HR $0.83,95 \%$ CI 0.49-1.39, Supplementary Table 2) that did not reach statistical significance.

Table 3 presents the association between pre-diagnostic cholesterol lowering drug use and all-cause mortality among breast cancer cases by stage of disease. For localized breast cancer, ever use of cholesterol lowering drugs had a $9 \%$ lower hazard for all-cause mortality (HR 0.91, 95\% CI 0.59-1.38) compared to never users. For regional/distant breast cancer, there was a $46 \%$ reduction in the hazard for
Table 3 Association between cholesterol lowering drug use and allcause mortality among women diagnosed with breast cancer in the MEC (2003-2014) by tumor stage

\begin{tabular}{llllll}
\hline & $n$ Cases & $n$ Deaths & HR & $95 \%$ CI & $p$ value \\
\hline $\begin{array}{l}\text { Localized } \\
\text { Never use (refer- } \\
\text { ence) }\end{array}$ & 578 & 56 & 1.00 & & \\
$\quad \begin{array}{l}\text { Ever use } \\
\text { Advanced }\end{array}$ & 488 & 60 & 0.91 & $0.59-1.38$ & 0.65 \\
$\begin{array}{l}\text { Never use (refer- } \\
\text { ence) }\end{array}$ & 207 & 65 & 1.00 & & \\
$\quad$\begin{tabular}{l} 
Ever use \\
\hline
\end{tabular} & 156 & 36 & $\mathbf{0 . 5 4}$ & $\mathbf{0 . 3 3 - 0 . 9 0}$ & $\mathbf{0 . 0 2}$ \\
\hline
\end{tabular}

Adjusted for age at breast cancer diagnosis, race/ethnicity, education, body mass index, daily caloric intake, Alternate Healthy Index Score, age at menarche, cardiovascular disease, diabetes, lymph node status, hormone receptor status, surgery, and hormone therapy. $p$-heterogeneity by stage $=0.48$

Bolded values indicate $p$-values $\leq 0.05$

${ }^{a}$ Advanced stage includes regional- and distant-stage tumors 
all-cause mortality (HR 0.54 95\% CI 0.33-0.90). However, there was no significant difference in the hazard ratios by stage ( $p$ for heterogeneity by stage $=0.48$ ).

For hormone receptor-positive breast cancer, ever use of cholesterol lowering drugs prior to diagnosis had a $31 \%$ lower hazard for all-cause mortality (HR 0.69, 95\% CI 0.48-0.99) compared to never user (Table 4). For hormone negative breast cancer, no association between cholesterol lowering drug use and mortality was observed. However, no evidence of differences between the hazard ratios was found ( $p$ for heterogeneity by hormone receptor status $=0.51$ ).

Supplementary Table 3 describes study characteristics of cholesterol lowering drug use across each racial/ethnic group. Native Hawaiian breast cancer cases (56\%) had the highest prevalence of ever use of cholesterol lowering drugs followed by Latino American (52\%), Japanese American (45\%), African American (39\%), and white (36\%) breast cancer cases. Age at breast cancer diagnosis was similar across racial/ethnic groups with the exception of a younger mean age of diagnosis among Native Hawaiians (70.8 years). Japanese American breast cancer cases had lower BMIs compared to the other racial/ethnic groups (56\% had normal BMI). While cardiovascular disease was similar across race/ethnicity, Latino American and Native Hawaiian breast cancer cases had a higher proportion of diabetes (29\% and $24 \%$, respectively). Across all racial/ethnic groups most women were likely to be diagnosed with localized breast cancer. African American breast cancer cases had a higher

Table 4 Association between cholesterol lowering drug use and allcause mortality among women diagnosed with breast cancer in the MEC (2003-2014) by hormone receptor status

\begin{tabular}{llllll}
\hline & $n$ Cases & $n$ Deaths & HR & $95 \%$ CI & $p$ value \\
\hline $\begin{array}{l}\text { Hormone receptor } \\
(+)^{\mathrm{a}}\end{array}$ & & & & & \\
$\begin{array}{l}\text { Never use (refer- } \\
\text { ence) }\end{array}$ & 656 & 83 & 1.00 & & \\
$\quad \begin{array}{l}\text { Ever use } \\
\text { Hormone receptor } \\
(-)^{\mathrm{a}}\end{array}$ & 544 & 71 & $\mathbf{0 . 6 9}$ & $\mathbf{0 . 4 8 - \mathbf { 0 . 9 9 }}$ & $\mathbf{0 . 0 5}$ \\
$\begin{array}{l}\text { Never use (refer- } \\
\text { ence) }\end{array}$ & 114 & 32 & 1.00 & & \\
$\quad$ Ever use & 86 & 23 & 1.04 & $0.52-2.07$ & 0.92 \\
\hline
\end{tabular}

Adjusted for, age at breast cancer diagnosis, race/ethnicity, education, body mass index, daily caloric intake, Alternate Healthy Index Score, age at menarche, cardiovascular disease, diabetes, tumor stage, lymph node status, surgery, and hormone therapy. $p$-heterogeneity by hormone receptor status $=0.51$

Bolded values indicate $p$-values $\leq 0.05$

${ }^{a}$ Hormone receptor $(+)$ includes tumors that are either estrogen receptor-positive or progesterone receptor-positive. Hormone receptor (-) includes tumors that are estrogen receptor and progesterone receptornegative proportion of hormone receptor negative breast cancer compared to other racial/ethnic groups (22\%).

Supplementary Table 4 presents the association between pre-diagnostic cholesterol lowering drug use and all-cause and breast cancer-specific mortality using the Inverse Probability of Treatment Weighting approach [28]. For all-cause mortality, ever use of cholesterol lowering drugs showed a $20 \%$ lowered hazard (HR $0.80,95 \%$ CI $0.67-0.95$ ) compared to never use. For breast cancer-specific mortality, a lowered hazard was observed for ever versus never use (HR 0.88 , 95\% CI 0.66-1.18) that was not statistically significant.

\section{Discussion}

In this multiethnic population, all-cause mortality was lower among women with breast cancer who reported having ever used cholesterol lowering drugs before diagnosis. Compared to never users, current users of cholesterol lowering drugs at assessment also experienced a significantly reduced all-cause mortality compared to never users, as did those who had used cholesterol lowering drugs for 3 years or less. Breast cancer specific-mortality was also suggested to be associated with pre-diagnostic cholesterol-lowering drug use. Women with advanced stage tumors had a lower all-cause mortality associated with ever use of cholesterol lowering drug use, compared to never users. In addition, women with hormone receptor-positive breast tumors had lower all-cause mortality associated with ever use of cholesterol lowering drug use.

While our questionnaire did not differentiate between the classes of cholesterol lowering drugs, we believe most of our cholesterol lowering drug users were taking statins, as statins have been the cornerstone of management for high blood cholesterol [29] since it was first approved by the Food and Drug Administration in 1987. Trends of statin therapy among adults since then have increased during the study period [30].

Our results of the associations of pre-diagnostic cholesterol lowering drug use with all-cause and, suggestively breast cancer-specific mortality agree with results from previous studies and meta-analyses [20,31]. A comprehensive meta-analysis by $\mathrm{Wu}$ et al. [20] reported a pooled result from five studies on statin use and breast cancer survival, showing a beneficial effect of pre-diagnostic statin use on overall survival (HR 0.68, 95\% CI 0.54-0.84) and on breast cancer-specific mortality (HR $0.7295 \%$ CI 0.53-0.99). A later metaanalysis by Liu et al. [31] also reported similar estimates on all-cause mortality (HR $0.72,95 \%$ CI $0.58-0.89$ ) and breast cancer specific-mortality (HR $0.73,95 \%$ CI $0.59-0.92$ ) based on seven studies.

Liu et al. [31] additionally performed a subgroup metaanalysis by follow-up time as a measure to assess duration 
of statin use. They found that longer follow-up time did not show a significant association between statin use and allcause mortality (HR 0.95, 95\% CI 0.75-1.19); yet less than 4 years of follow-up showed a protective effect of statins against all-cause mortality (HR 0.61, 95\% CI 0.45-0.80). A more recent cohort study by Li et al. [25] found that statin use more than 5 years was associated with improved overall survival (HR 0.38, 95\% CI 0.17-0.85) compared to use less than 3 years (HR 1.50, 95\% CI 0.79-2.86) and between 3 and 5 years (HR 1.07, 95\% CI 0.52-2.15). In our study, cholesterol-lowering drug use for 3 years or less was associated with lower all-cause mortality in comparison to never use. Due to limited sample size, we were unable to evaluate a larger range in the duration of cholesterol-lowering drug use or to evaluate breast cancerspecific mortality. A possible reason for the differences in hazard ratios by length of use in our cohort of cases may be due to the progression of cardiovascular symptoms over time, increasing the risk for all-cause mortality among cases with longer duration of use.

Murtola et al. [22] reported a HR of 0.58 (95\% CI 0.49-0.70) among current users of statins associated with localized breast cancer versus statin never users, while current users among women with metastatic breast cancer had a HR of 0.66 (95\% CI 0.47-0.92) versus statin never users. Our study found cholesterol lowering drug use reduced all-cause mortality among women with advanced stage (regional and distant) tumors compared to never users. No statistically significant reduction in mortality was seen among cholesterol lowering drug users with localized tumors in our cohort.

Differences in the associations between cholesterol lowering drug use and mortality among breast cancer cases by hormone receptor status has been investigated in some studies [16, 18, 24]. McMenamin et al. [16] found borderline statistically significant evidence of reduced overall mortality in a subgroup analysis of 1495 women with ER-positive breast tumors, who had ever used statins (HR 0.85, 95\% CI 0.72, 1.01). Our findings for hormone receptor-positive breast cancers suggest beneficial effects on overall survival from cholesterol lowering drug use as reported in other studies.

A possible biological mechanism by which cholesterol lowering drug use influences overall mortality may be due to the inhibition of the mevalonate pathway to reduce cholesterol synthesis that is relevant for multiple pathways for cholesterol-related diseases. Specifically for the reduced risk for hormone receptor-positive breast cancer mortality, cholesterol is the building block for hormones (e.g. estrogen and progesterone) and the recently identified selective endogenous estrogen receptor modulator (SERM), 27-hydroxycholesterol; therefore, inhibition of cholesterol synthesis may result in reduced cellular proliferation for hormone receptorpositive breast tumors [32].
A study by the Breast International Group based on clinical trial data of 7963 postmenopausal women with early stage hormone receptor-positive invasive breast cancer showed that initiation of cholesterol-lowering medications during any endocrine therapy was associated with improved disease-free survival compared to never users (HR 0.79, 95\% CI 0.66-0.95) [33]. Shaitelman et al. [24] conducted a study of statin use among triple-negative (ERnegative, PR-negative, HER2-negative) breast cancer and found no significant association between statin use and overall survival (HR 0.74, 95\% CI 0.20-2.77).

Reports on the use of cholesterol lowering drug across race/ethnicity for breast cancer survivors are sparse. Similar to Leiter et al. [23], our study found similar prevalence of cholesterol lowering drug use between African American and white breast cancer cases. Li et al. [25] and Shaitelman et al. [24] also reported similar prevalence of use among African American cases as our study (39\% ever users in all three studies). Our study is the first to report a high prevalence of cholesterol lower drug use among Japanese American (53\% ever users) and Native Hawaiian (56\% ever users) breast cancer cases compared to White cases (37\% ever users).

Our study has limitations to consider. It was based on the assessment of cholesterol lowering drug use at a single time point prior to breast cancer diagnosis with possible misclassification of exposure; yet we would expect this misclassification to be non-differential by vital status. Information on duration and specific types of cholesterol lowering drug use was limited to specific time categories and we were unable to evaluate differences in duration by more refined years due to limited detailed data from our questionnaire. Due to limited sample size and deaths by race/ethnicity, we were unable to examine racial/ethnicspecific associations for breast cancer specific mortality.

As one of the most commonly occurring cancers worldwide, breast cancer is one of the most researched carcinomas. As a result, the survival of women with breast cancer has improved significantly over the past few decades. Yet, continued research is needed to address the persistent disparities in survival, especially disparities that may arise due to modifiable factors associated with race and ethnicity, such as barriers to healthcare, health beliefs and behaviors, and socioeconomic mobility. Established drugs such as cholesterol lowering drugs may be a means to improve survival outcomes, while also being a safer alternative compared to newer drugs with its well-known safety profile. Multiethnic data are a valuable resource to evaluate survival outcomes across racial/ethnic groups. 


\section{Conclusion}

In this multiethnic population of breast cancer cases, use of cholesterol lowering drugs reduced all-cause mortality compared to never use. The results from our study are promising as they confirm results found in literature among a multiethnic population. Future studies should examine the effects of post-diagnostic use, time-dependent use and type of cholesterol lowering drug use on breast cancer survival outcomes across racially/ethnically diverse populations.

Supplementary Information The online version contains supplementary material available at https://doi.org/10.1007/s10549-021-06360-y.

Author contributions NM conducted all data analysis, interpreted the data, and led the writing the manuscript. IC and LRW led the study design. All authors assisted in interpreting the results of the data in their review of the manuscript. IC, LWML, LLM, LRW, JY and CYH were major contributors in reviewing and editing the manuscript. All authors read, reviewed, and approved the final manuscript.

Funding This research was supported by the National Cancer Institute (U01 CA164973, R01CA229815).

Data availability The data that support the findings of this study are available to researchers through the procedures described at https:// www.uhcancercenter.org/for-researchers/mec-data-sharing.

\section{Declarations}

Conflict of interest The authors declare no conflict of interests.

Ethical approval This study was conducted according to the guidelines laid down in the Declaration of Helsinki and all procedures involving human subjects/patients were approved by the Committee on Human Studies at the University of California, San Francisco, University of Hawaii, and the University of Southern California. The study protocol was approved by the Institutional Review Boards of the University of California, San Francisco, University of Hawaii, and the University of Southern California.

Informed consent Subjects consented to participate upon receipt of completed baseline questionnaire.

Open Access This article is licensed under a Creative Commons Attribution 4.0 International License, which permits use, sharing, adaptation, distribution and reproduction in any medium or format, as long as you give appropriate credit to the original author(s) and the source, provide a link to the Creative Commons licence, and indicate if changes were made. The images or other third party material in this article are included in the article's Creative Commons licence, unless indicated otherwise in a credit line to the material. If material is not included in the article's Creative Commons licence and your intended use is not permitted by statutory regulation or exceeds the permitted use, you will need to obtain permission directly from the copyright holder. To view a copy of this licence, visit http://creativecommons.org/licenses/by/4.0/.

\section{References}

1. Holmes MD, Chen WY (2012) Hiding in plain view: the potential for commonly used drugs to reduce breast cancer mortality. Breast Cancer Res 14:216. https://doi.org/10.1186/bcr3336

2. Prochilo T, Di Biasi B, Aroldi F et al (2014) Blind snipers: relevant off target effects of non-chemotherapeutic agents in oncology: review of the literature. Rev Recent Clin Trials 9:102-114

3. Boguski MS, Mandl KD, Sukhatme VP (2009) Repurposing with a difference. Science 324:1394-1395. https://doi.org/10.1126/ science. 1169920

4. Ashburn TT, Thor KB (2004) Drug repositioning: identifying and developing new uses for existing drugs. Nat Rev Drug Discov 3:673-683. https://doi.org/10.1038/nrd1468

5. Sehdev A, O'Neil BH (2015) The role of aspirin, vitamin D, exercise, diet, statins, and metformin in the prevention and treatment of colorectal cancer. Curr Treat Options Oncol 16:43. https://doi.org/10.1007/s11864-015-0359-z

6. Holmes MD, Hankinson SE, Feskanich D, Chen WY (2013) Beta blockers and angiotensin converting enzyme inhibitors' purported benefit on breast cancer survival may be explained by aspirin use. Breast Cancer Res Treat 139:507-513. https:// doi.org/10.1007/s10549-013-2553-7

7. Stopsack KH, Greenberg AJ, Mucci LA (2017) Common medications and prostate cancer mortality: a review. World J Urol 35:875-882. https://doi.org/10.1007/s00345-016-1912-5

8. Rao S, Lowe M, Herliczek TW, Keyomarsi K (1998) Lovastatin mediated G1 arrest in normal and tumor breast cells is through inhibition of CDK2 activity and redistribution of p21 and p27, independent of p53. Oncogene 17:2393-2402. https://doi.org/ 10.1038/sj.onc. 1202322

9. Jakobisiak M, Golab J (2003) Potential antitumor effects of statins (review). Int J Oncol 23:1055-1069. https://doi.org/10. 3892/ijo.23.4.1055

10. Fritz G (2005) HMG-CoA reductase inhibitors (statins) as anticancer drugs (review). Int J Oncol 27:1401-1409

11. Warita K, Warita T, Beckwitt CH et al (2014) Statin-induced mevalonate pathway inhibition attenuates the growth of mesenchymal-like cancer cells that lack functional E-cadherin mediated cell cohesion. Sci Rep 4:7593. https://doi.org/10.1038/ srep07593

12. Karlic H, Thaler R, Gerner $\mathrm{C}$ et al (2015) Inhibition of the mevalonate pathway affects epigenetic regulation in cancer cells. Cancer Genet 208:241-252. https://doi.org/10.1016/j.cancergen.2015. 03.008

13. Di Bello E, Zwergel C, Mai A, Valente S (2020) The innovative potential of statins in cancer: new targets for new therapies. Front Chem. https://doi.org/10.3389/fchem.2020.00516

14. Mullen PJ, Yu R, Longo J et al (2016) The interplay between cell signalling and the mevalonate pathway in cancer. Nat Rev Cancer 16:718-731. https://doi.org/10.1038/nrc.2016.76

15. Cardwell CR, Hicks BM, Hughes C, Murray LJ (2015) Statin use after diagnosis of breast cancer and survival: a population-based cohort study. Epidemiology 26:68-78. https://doi.org/10.1097/ EDE.0000000000000189

16. Mc Menamin UC, Murray LJ, Hughes CM, Cardwell CR (2016) Statin use and breast cancer survival: a nationwide cohort study in Scotland. BMC Cancer 16:600. https://doi.org/10.1186/ s12885-016-2651-0

17. Smith A, Murphy L, Sharp L et al (2016) De novo post-diagnosis statin use, breast cancer-specific and overall mortality in women with stage I-III breast cancer. Br J Cancer 115:592-598. https:// doi.org/10.1038/bjc.2016.232 
18. Smith A, Murphy L, Zgaga L et al (2017) Pre-diagnostic statin use, lymph node status and mortality in women with stages. Br J Cancer 117:588-596. https://doi.org/10.1038/bjc.2017.227

19. Borgquist S, Broberg P, Tojjar J, Olsson H (2019) Statin use and breast cancer survival-a Swedish nationwide study. BMC Cancer. https://doi.org/10.1186/s12885-018-5263-z

20. Wu Q-J, Tu C, Li Y-Y et al (2015) Statin use and breast cancer survival and risk: a systematic review and meta-analysis. Oncotarget 6:42988-43004. https://doi.org/10.18632/oncotarget.5557

21. Nickels S, Vrieling A, Seibold P et al (2013) Mortality and recurrence risk in relation to the use of lipid-lowering drugs in a prospective breast cancer patient cohort. PLoS ONE 8:e75088. https://doi.org/10.1371/journal.pone.0075088

22. Murtola TJ, Visvanathan K, Artama M et al (2014) Statin use and breast cancer survival: a nationwide cohort study from Finland. PLoS ONE 9:e110231. https://doi.org/10.1371/journal.pone. 0110231

23. Leiter A, Bickell NA, LeRoith D et al (2018) Statin use and breast cancer prognosis in black and white women. Horm Cancer 9:5561. https://doi.org/10.1007/s12672-017-0312-7

24. Shaitelman SF, Stauder MC, Allen P et al (2017) Impact of statin use on outcomes in triple negative breast cancer. J Cancer 8:2026-2032. https://doi.org/10.7150/jca.18743

25. Li YR, Ro V, Steel L et al (2019) Impact of long-term lipidlowering therapy on clinical outcomes in breast cancer. Breast Cancer Res Treat 176:669-677. https://doi.org/10.1007/ s10549-019-05267-z

26. Kolonel LN, Henderson BE, Hankin JH et al (2000) A Multiethnic Cohort in Hawaii and Los Angeles: baseline characteristics. Am J Epidemiol 151:346-357

27. Harmon BE, Boushey CJ, Shvetsov YB et al (2015) Associations of key diet-quality indexes with mortality in the Multiethnic
Cohort: the Dietary Patterns Methods Project12345. Am J Clin Nutr 101:587-597. https://doi.org/10.3945/ajcn.114.090688

28. Austin PC (2011) An introduction to propensity score methods for reducing the effects of confounding in observational studies. Multivariate Behav Res 46:399-424. https://doi.org/10.1080/00273 171.2011.568786

29. Jones PH (2004) Statins as the cornerstone of drug therapy for dyslipidemia: monotherapy and combination therapy options. Am Heart J 148:S9-S13. https://doi.org/10.1016/j.ahj.2004.04.026

30. Sarpong EM, Zuvekas SH. Trends in Statin Therapy among Adults (Age $\geq 18$ ), United States, 2000 to 2011. Statistical Brief \#458. November 2014. Agency for Healthcare Research and Quality, Rockville, MD

31. Liu B, Yi Z, Guan X et al (2017) The relationship between statins and breast cancer prognosis varies by statin type and exposure time: a meta-analysis. Breast Cancer Res Treat 164:1-11. https:// doi.org/10.1007/s10549-017-4246-0

32. Kimbung S, Lettiero B, Feldt $M$ et al (2016) High expression of cholesterol biosynthesis genes is associated with resistance to statin treatment and inferior survival in breast cancer. Oncotarget 7:59640-59651. https://doi.org/10.18632/oncotarget.10746

33. Borgquist S, Giobbie-Hurder A, Ahern TP et al (2017) Cholesterol, cholesterol-lowering medication use, and breast cancer outcome in the BIG 1-98 Study. J Clin Oncol 35:1179-1188. https:// doi.org/10.1200/JCO.2016.70.3116

Publisher's Note Springer Nature remains neutral with regard to jurisdictional claims in published maps and institutional affiliations. 